\title{
Minimal gaugomaly mediation
}

\section{Yi Cai and Markus A. Luty}

Physics Department, University of California, One Shields Avenue, Davis CA, U.S.A.

E-mail: caiyi.pku@gmail.com, markusluty@gmail.com

ABSTRACT: Mixed anomaly and gauge mediation ("gaugomaly" mediation) gives a natural solution to the SUSY flavor problem with a conventional LSP dark matter candidate. We present a minimal version of gaugomaly mediation where the messenger masses arise directly from anomaly mediation, automatically generating a messenger scale of order $50 \mathrm{TeV}$. We also describe a simple relaxation mechanism that gives rise to realistic $\mu$ and $B \mu$ terms. $B$ is naturally dominated by the anomaly-mediated contribution from top loops, so the $\mu-B \mu$ sector only depends on a single new parameter. In the minimal version of this scenario the full SUSY spectrum is determined by two continuous parameters (the anomaly- and gauge-mediated SUSY breaking masses) and one discrete parameter (the number of messengers). We show that these simple models can give realistic spectra with viable dark matter.

KEYwORDS: Supersymmetry Phenomenology

ARXiv EPRINT: 1008.2024 


\section{Contents}

1 Introduction 1

2 The messenger threshold $\quad 2$

3 The $\mu-B \mu$ sector 5

4 Phenomenology 8

4.1 Spectrum 8

4.2 Dark matter 9

4.3 Fine tuning 9

5 Conclusions $\quad 11$

$\begin{array}{ll}\text { A SUSY breaking } & 12\end{array}$

\section{Introduction}

Anomaly-mediated SUSY breaking (AMSB) is a natural solution to the flavor problem $[1,2]$ that may be naturally realized in string theory [3]. The minimal model with only the MSSM in the visible sector predicts negative slepton masses. However, ref. [4] pointed out that if the visible sector contains messenger fields whose masses arise from AMSB, they automatically give messenger masses with $F / M \sim F_{\varphi}$, where $F_{\varphi} \sim 50 \mathrm{TeV}$ is the anomaly-mediated order parameter. This means that the gauge- and anomaly-mediated contributions are automatically of the same size, and this kind of model easily give a realistic SUSY breaking spectrum. We call this "gaugomaly" mediation, following ref. [5]. An important difference from gauge mediation is that the gravitino is naturally heavy (of order $F_{\varphi}$ ) so the LSP is generally a neutralino, and therefore a WIMP dark matter candidate. Gaugomaly mediation is probably the simplest framework for SUSY breaking that solves the SUSY flavor problem and has a natural dark matter candidate.

It was pointed out in ref. [6] that messenger masses can arise from holomorphic Kähler terms of the form $\int d^{4} \theta \bar{\Phi} \Phi+$ h.c. If the dimensionless coefficient of such a term is order 1 this gives a messenger threshold at the scale $F_{\varphi}$. Unfortunately slepton masses remain negative at the messenger scale in this model. Running from the scale $F_{\varphi}$ down to the weak scale can give positive slepton masses from the enhanced gaugino contributions to the scalar masses for a sufficiently large threshold correction, but this requires a large number of messengers and additional fine-tuning. Ref. [7] showed that a successful model can easily be obtained with additional singlet fields. If these have order 1 holomorphic Kähler terms they naturally get SUSY breaking VEVs set by the scale $F_{\varphi}$. Coupling these 
fields to messengers then gives a messenger threshold that can give an acceptable spectrum with positive slepton masses at the messenger threshold.

In this paper, we expand and improve upon ref. [7] in several ways. First, we construct simple models that generate $\mu$ and $B \mu$ terms of the right size at the messenger threshold. Second, we compute the SUSY spectrum and show that there are solutions with viable WIMP dark matter. We also include a discussion of tadpole terms for singlet fields that were neglected in ref. [7] (but do not change the results), and give complete formulas for the SUSY breaking masses to all orders in $F / M^{2}$.

The models we present are technically natural, but some superpotential couplings allowed by symmetries are absent. There are good reasons to think that technical naturalness may well be all that we should expect in supersymmetric theories. For example, if superpotential couplings arise from the VEVs are chiral superfields, then any technically natural theory is the most general theory invariant under a set of symmetries, some of which are broken by the VEVs [8]. The mechanisms at work in our models are simple and robust, and it is likely that they work in models that include all terms allowed by symmetries. Focusing on simple technically natural models allows us to illustrate the basic mechanism in the simplest possible setting.

The general picture that emerges from this work is the following. In AMSB the scale $F_{\varphi}$ is a natural scale for singlets and vector-like matter to get masses and/or VEVs. Such additional fields do not ruin gauge coupling unification, and are ubiquitous in string constructions. One may therefore expect many such fields with masses of order $F_{\varphi}$. Some of these fields act as messengers, and the spectrum will interpolate between an anomalymediated spectrum and a gauge-mediated spectrum. This is a rather simple and appealing picture that accords well with general theoretical expectations.

It also gives a very predictive spectrum of SUSY breaking masses. The $\mu$ and $B \mu$ terms are generated via a relaxation mechanism, but quite generally the contribution to the $B$ term from this sector is smaller than the anomaly-mediated contribution, which comes from a top loop. Therefore, the $\mu-B \mu$ sector effectively contributes a single free parameter, namely the value of $\mu$. The SUSY breaking spectrum is then specified by $F_{\varphi}$, a gauge mediated order parameter $F / M \sim F_{\varphi}, \mu$, and the number of messengers $N$. There is a weak dependence on the ratio $x=F / M^{2}$ that is unimportant except near $x=1$. Once we fix the Higgs VEV to its experimental value, the minimal model depends on only two continuous parameters, and the number of messengers. We find regions of this parameter space where the dark matter relic abundance has the correct value, and dark matter direct detection is below current bounds.

\section{The messenger threshold}

We now show that a realistic messenger threshold at $F_{\varphi} \sim 50 \mathrm{TeV}$ arises naturally in simple anomaly-mediated models. We include a UV divergent tadpole contribution that was neglected in ref. [7] that does not change the main conclusions. 
The basic observation $[6]$ is that $F_{\varphi} \neq 0$ generates tree-level mass terms via holomorphic Kähler terms of the form

$$
\begin{aligned}
\Delta \mathcal{L} & =\int d^{4} \theta \frac{\varphi^{\dagger}}{\varphi} c \bar{\Phi} \Phi+\text { h.c. } \\
& =c F_{\varphi}^{\dagger} \int d^{2} \theta \varphi^{-1} \bar{\Phi} \Phi+\text { h.c. }
\end{aligned}
$$

Here $c$ is a dimensionless constant and $\bar{\Phi}, \Phi$ are vectorlike chiral fields.

Holomorphic Kähler terms may not be familiar, but arise naturally from integrating out heavy states at tree level. For example, consider the coupling of $\bar{\Phi} \Phi$ to heavy singlet fields $\Sigma, \bar{\Sigma}$ via

$$
\Delta W=\kappa \varphi^{2} \Sigma+M \varphi \bar{\Sigma} \Sigma+\frac{1}{2} \lambda \Sigma \bar{\Sigma}^{2}+y \Sigma \bar{\Phi} \Phi
$$

This has a $\mathrm{U}(1)_{R}$ symmetry with

$$
R(\Sigma)=2, \quad R(\bar{\Sigma})=R(\bar{\Phi} \Phi)=0 .
$$

Note that the $\mathrm{U}(1)_{R}$ symmetry eq. (2.4) allows the holomorphic Kähler term but forbids a superpotential mass term $\int d^{2} \theta \bar{\Phi} \Phi$. We now show that such a term is generated with an order-1 coefficient if $\kappa \sim M^{2}$ and $\lambda, y \sim 1$. The VEVs are

$$
\langle\Sigma\rangle=0, \quad\langle\bar{\Sigma}\rangle=\frac{-M \pm \sqrt{M^{2}-2 \lambda \kappa}}{\lambda} .
$$

The messengers are therefore massless at this order, while $\Sigma, \bar{\Sigma}$ have masses of order $M$. Integrating out $\Sigma, \bar{\Sigma}$ gives a vanishing superpotential and a holomorphic Kähler term of the form eq. (2.1) with

$$
c=\left(-1 \pm \frac{M}{\sqrt{M^{2}-2 \lambda \kappa}}\right) \frac{y}{\lambda} .
$$

Here we assumed that all couplings are real, and $M^{2}-2 \lambda \kappa>0$. We conclude that holomorphic Kähler terms are generically present when allowed by symmetries.

To understand the effect of holomorphic Kähler terms like eq. (2.1) on the SUSY spectrum, note that the messenger threshold has $F / M=-F_{\varphi}$. For comparison, a superpotential mass term $\int d^{2} \theta M \varphi \bar{\Phi} \Phi$ with $F / M=+F_{\varphi}$ gives threshold corrections that put the SUSY breaking terms on the anomaly mediated renormalization group trajectory in the low-energy effective theory. (There are additional corrections of order $F / M^{2}=1 / c \sim 1$, but in practice these corrections are small unless $F / M^{2}=1$ to high accuracy; see the appendix.) If $\bar{\Phi}, \Phi$ are messengers, the term eq. (2.1) gives a threshold correction that affects the scalar masses like a supersymmetric mass, since these are even in $F / M$. Therefore the slepton masses are negative below the messenger threshold. The gaugino masses are odd in $F / M$ and receive a nontrivial correction at threshold. Running from the messenger scale to the weak scale can give positive slepton masses, but this requires a large number of messengers and results in a very fine-tuned model [6].

These problems can be easily cured if the messenger threshold includes singlets in addition to messengers [7]. These singlets naturally get SUSY-breaking VEVs of order 
$F_{\varphi}$, and couplings between the singlets and the messengers naturally give large threshold corrections to scalar masses that can give a natural spectrum. A simple example consists of a singlet $X$, and doublet and triplet messengers $\bar{D}, D$ and $\bar{T}, T$, with all dimensionless couplings:

$$
\begin{aligned}
& K=-\frac{\varphi^{\dagger}}{\varphi}\left(\frac{1}{2} c_{X} X^{2}+c_{D} \bar{D} D+c_{T} \bar{T} T\right), \\
& W=\frac{\lambda_{X}}{3 !} X^{3}+\lambda_{D} X \bar{D} D+\lambda_{T} X \bar{T} T .
\end{aligned}
$$

This theory is only technically natural, since a linear superpotential term $\Delta W \sim \varphi^{2} X$ is allowed by all symmetries. ${ }^{1}$

Note that there is a UV divergent 1-loop linear term in $X$ :

$$
\begin{aligned}
\Delta \mathcal{L}_{\text {eff }}= & \int d^{4} \theta\left[-\frac{c \lambda F_{\varphi}}{16 \pi^{2}}\left(\phi^{-1}\right)^{\dagger} \ln \frac{\Lambda|\varphi|}{\mu} X+\text { h.c. }\right] \\
= & \frac{c \lambda F_{\varphi}^{2}}{16 \pi^{2}}\left(\ln \frac{\Lambda}{\mu}-\frac{1}{2}\right) \int d^{2} \theta X+\text { h.c. } \\
& +\frac{c \lambda F_{\varphi}^{3}}{32 \pi^{2}} X+\text { h.c. }
\end{aligned}
$$

where

$$
c \lambda \equiv c_{X} \lambda_{X}+c_{D} \lambda_{D}+c_{T} \lambda_{T}
$$

The UV divergent $\int d^{2} \theta$ term tells us that we have an uncalculable coupling

$$
\Delta W=\kappa_{X} X
$$

Note that there is no $\varphi$ dependence in this term, since it arises from the SUSY breaking from $F_{\varphi} \neq 0$ as described above. The coupling $\kappa_{X}$ has the renormalization group equation

$$
\frac{d \kappa_{X}}{d \ln \mu}=-\frac{c \lambda F_{\varphi}^{2}}{16 \pi^{2}}-\frac{1}{2} \kappa_{X} \gamma_{X}
$$

where $\gamma_{X}=d \ln Z_{X} / d \ln \mu$ is the wavefunction renormalization of $X$. If we run down from a large scale $\Lambda$ (e.g. the GUT scale) the large logs compensate for the loop suppression, and we expect

$$
\kappa_{X} \sim-\frac{c \lambda F_{\varphi}^{2}}{16 \pi^{2}} \ln \frac{\Lambda}{F_{\varphi}} \sim-c \lambda F_{\varphi}^{2} .
$$

The model is therefore described by the effective superpotential

$$
\begin{aligned}
W_{\mathrm{eff}}= & -a_{X} F_{\varphi}^{2} X-F_{\varphi} \varphi^{-1}\left(\frac{1}{2} c_{X} X^{2}+c_{D} \bar{D} D+c_{T} \bar{T} T\right) \\
& +\frac{\lambda_{X}}{3 !} X^{3}+\lambda_{D} X \bar{D} D+\lambda_{T} X \bar{T} T .
\end{aligned}
$$

with $a_{X} \sim+c \lambda$. We have neglected the finite tadpole in eq. (2.10), since it is loop suppressed (and not log enhanced). Including it will give a 1-loop correction to the VEVs

\footnotetext{
${ }^{1}$ A superpotential term $\Delta W \sim \varphi X^{2}$ can be forbidden by a discrete $R$ symmetry $X(\theta) \mapsto-X(i \theta)$.
} 
and masses of the particles. All SUSY breaking is then in the mass terms, which are all of order $F_{\varphi}$. The potential has a tadpole term for $X$

$$
\begin{aligned}
V= & a_{X} c_{X} X-\frac{1}{2}\left(c_{X}-\lambda_{X} a_{X}\right) X^{2}+\text { h.c. } \\
& +c_{X}^{2}|X|^{2}+\mathcal{O}\left(X^{3}\right)
\end{aligned}
$$

and therefore $\langle X\rangle \neq 0$. For example, for $\left|a_{X}\right| \ll 1$ and $\left|c_{X}\right|>1$ the VEVs are dominated by the linear and quadratic terms in the potential and we obtain a stable minimum with

$$
\langle X\rangle=-\frac{a_{X}}{c_{X}-1} F_{\varphi}, \quad\left\langle F_{X}\right\rangle=\frac{\left(2 c_{X}-3\right) a_{X}^{2}}{2\left(c_{X}-1\right)^{2}} F_{\varphi}^{2}
$$

Exact expressions for the case $a_{X}=0$ can be found in ref. [7]. The important point is that the coupling of $X$ to the messengers gives them a general value of $F / M \sim F_{\varphi}$.

In a grand unified theory it is natural to impose boundary conditions at the unification scale

$$
c_{D}\left(M_{\mathrm{GUT}}\right)=c_{T}\left(M_{\mathrm{GUT}}\right), \quad \lambda_{D}\left(M_{\mathrm{GUT}}\right)=\lambda_{T}\left(M_{\mathrm{GUT}}\right) .
$$

Since all couplings run only due to wavefunction renormalization, we have

$$
\frac{d}{d \ln \mu}\left(\frac{c_{D, T}}{\lambda_{D, T}}\right)=\frac{1}{2} \gamma_{X} \frac{c_{D, T}}{\lambda_{D, T}}
$$

which implies that

$$
\frac{c_{D}}{\lambda_{D}}=\frac{c_{T}}{\lambda_{T}} \equiv s
$$

at all scales. In this case, the messenger threshold for doublets and singlets are controlled by the same SUSY breaking parameter. Writing the messenger masses as

$$
W_{\mathrm{eff}}=M_{D} \bar{D} D+M_{T} \bar{T} T
$$

we have

$$
\frac{F}{M}=\frac{F_{M_{T}}}{M_{T}}=\frac{\left\langle F_{X}\right\rangle-s\left|F_{\varphi}\right|^{2}}{\langle X\rangle+s F_{\varphi}^{\dagger}} .
$$

In this model, the SUSY breaking spectrum (excluding the $\mu$ and $B \mu$ terms; see below) is parameterized by $F_{\varphi}$ and $F / M$. The details are given in the appendix, including a derivation of the results to all orders in $F / M^{2}$. This is in principle important because $F / M^{2} \sim 1$ (since all mass terms are of order $F_{\varphi}$ ), but in practice the higher order corrections are numerically negligible unless $F / M^{2}=1$ to high accuracy.

The model described above does not address the $\mu$ problem. We will see that a realistic model of the $\mu$ term requires only a modest complication of the threshold at the scale $F_{\varphi}$.

\section{The $\mu-B \mu$ sector}

Adding a supersymmetric $\mu$ term to the Lagrangian in AMSB gives $B \mu=F_{\varphi} \mu$, which generates a Higgs VEV of order $F_{\varphi} \sim 50 \mathrm{TeV}$. This means that the $\mu$ term (or better, the 
Higgsino mass term) must originate in some other way in AMSB to avoid a phenomenological disaster. We present a simple relaxation mechanism to suppress $B \mu$. Our model requires a small coupling of order $10^{-2}$ to suppress $\mu$ relative to the messenger scale, but with this one small parameter $B \mu$ automatically has the correct size.

The model has two additional singlet superfields fields $S$ and $A$ with superpotential

$$
\Delta W=-a F_{\varphi}^{2} A+\frac{1}{2} \lambda A S^{2}+\epsilon S H_{u} H_{d}
$$

and Kähler potential

$$
\Delta K=-\frac{\varphi^{\dagger}}{\varphi}\left(\frac{1}{2} c S^{2}\right)
$$

The linear term in eq. (3.1) is required due to the UV divergent tadpole, as discussed in the previous section, and we expect $a \sim+c \lambda$. A linear term in $S$ is forbidden by the $Z_{2}$ symmetry

$$
S \mapsto-S, \quad H_{u} H_{d} \mapsto-H_{u} H_{d},
$$

with all other fields even.

Note that $A$ appears in the tree-level scalar potential only via the $\left|F_{S}\right|^{2}$ term, so minimizing the potential with respect to $A$ implies $\left\langle F_{S}\right\rangle=0$. This model therefore generates a tree-level $\mu$ term without a tree-level $B \mu$ term. This is a relaxation mechanism for the $B \mu$ term similar to that of ref. [9]. The correct size of the $\mu$ term is obtained for $\epsilon \sim 10^{-2}$, and a $B \mu$ term of order $\epsilon F_{\varphi} / 16 \pi^{2}$ is generated at one loop. All components of the fields $A$ and $S$ have masses of order $F_{\varphi}$ (assuming that $\lambda, c \sim 1$ ), so the theory below the scale $F_{\varphi}$ is the MSSM.

The smallness of $\epsilon$ is perfectly natural since it is the only coupling that violates the discrete symmetry $S \mapsto-S$, with all other fields even. On the other hand, the model taken as a whole is only technically natural, since a superpotential term $\Delta W \sim \varphi^{2} A$ is allowed by all symmetries, and would ruin the relaxation mechanism described above. ${ }^{2}$

We now analyze the model in detail. The tree-level potential is

$$
\begin{aligned}
V= & \left|\lambda A S-c F_{\varphi} S+\epsilon H_{u} H_{d}\right|^{2}+\left|\frac{1}{2} \lambda S^{2}-a F_{\varphi}^{2}\right|^{2}+\epsilon^{2}|S|^{2}\left(\left|H_{u}\right|^{2}+\left|H_{d}\right|^{2}\right) \\
& -\frac{1}{2} c F_{\varphi}^{2} S^{2}+\text { h.c. }
\end{aligned}
$$

We minimize with respect to $S$ and $A$, expanding about $H_{u}, H_{d}=0$. This gives

$$
\begin{aligned}
\langle A\rangle & =\frac{c F_{\varphi}}{\lambda}, \\
\langle S\rangle & = \pm \frac{\sqrt{2(c+a \lambda)} F_{\varphi}}{\lambda},
\end{aligned}
$$

assuming that $c+a \lambda>0$. Note that $\left\langle F_{S}\right\rangle=0$ due to the $A$ minimization condition, so we obtain

$$
\mu=\epsilon\langle S\rangle, \quad B \mu=0 .
$$

The minimum spontaneously breaks the discrete symmetry eq. (3.3), so this theory has domain walls. These can be eliminated by adding additional naturally small couplings (e.g.

\footnotetext{
${ }^{2}$ Superpotential terms of the form $\Delta W \sim \varphi A^{2}+\varphi S^{2}+A^{3}$ can be forbidden by a $\mathrm{U}(1)_{R}$ symmetry.
} 
higher-dimension operators) that break this symmetry. All scalar and fermion components of $S$ and $A$ get masses of order $F_{\varphi}$. These masses are all equal to $\lambda\langle S\rangle$, suggesting a hidden unbroken SUSY in the model.

The hidden SUSY can be made manifest by a superfield field redefinition

$$
A=A^{\prime}-\frac{c F_{\varphi}}{\lambda \varphi}
$$

We then have

$$
\int d^{4} \theta A^{\dagger} A=\int d^{2} \theta A^{\prime \dagger} A^{\prime}-\left(\int d^{2} \theta \frac{c F_{\varphi}^{2}}{\lambda} A^{\prime}+\text { h.c. }\right)+\text { constant. }
$$

The effective superpotential is therefore

$$
W_{\text {eff }}=-\left(a+\frac{c}{\lambda}\right) F_{\varphi}^{2} A^{\prime}+\frac{1}{2} \lambda A^{\prime} S^{2}+\epsilon S H_{u} H_{d}
$$

There is no conformal compensator in the linear term because it arises from the couplings eqs. (3.1) and (3.2), so the Lagrangian preserves SUSY at tree level. We see that the VEVs eq. (3.6) are simply the supersymmetric vacuum $\left\langle F_{S}\right\rangle=\left\langle F_{A^{\prime}}\right\rangle=0$. Expanding about this vacuum $S=\langle S\rangle+S^{\prime}$ gives a superpotential

$$
W_{\mathrm{eff}}=\lambda\langle S\rangle A^{\prime} S^{\prime}+\frac{1}{2} \lambda A^{\prime} S^{\prime 2}+\cdots
$$

This explains why all components of $A$ and $S$ have a mass equal to $\lambda\langle S\rangle$. The hidden supersymmetry of this model is rather special. The relaxation mechanism does not depend on this, and more complicated models will not necessarily have this structure.

We now discuss the $B \mu$ term. As we have seen above, it vanishes at tree-level, but there are nonvanishing 1-loop contributions. From eq. (3.10) we see that the tree-level potential is actually supersymmetric. The only SUSY breaking comes from the finite linear term arising from the finite part of the $A$ tadpole (see eq. (2.10)). In terms of the shifted fields $A^{\prime}$ and $S^{\prime}$ we have

$$
V=-\frac{c \lambda F_{\varphi}^{3}}{32 \pi^{2}}\left(A^{\prime}+\text { h.c. }\right)+|\lambda\langle S\rangle|^{2}\left(\left|A^{\prime}\right|^{2}+\left|S^{\prime}\right|^{2}\right)+\cdots
$$

We therefore obtain

$$
\left\langle A^{\prime}\right\rangle=\frac{1}{64 \pi^{2}} \frac{c \lambda}{c+a \lambda} F_{\varphi}
$$

The $B \mu$ term is therefore

$$
B \mu=\epsilon \lambda\left\langle A^{\prime}\right\rangle\langle S\rangle+B_{\mathrm{AMSB}} \mu=\left(\lambda\left\langle A^{\prime}\right\rangle+B_{\mathrm{AMSB}}\right) \mu,
$$

where

$$
B_{\mathrm{AMSB}}=\frac{1}{2}\left(\gamma_{H_{u}}+\gamma_{H_{d}}\right) F_{\varphi}
$$

Using

$$
\gamma_{H_{u}}=-\frac{N_{c} y_{t}^{2}}{8 \pi^{2}}+\cdots
$$


where $N_{c}=3$ is the number of colors, the contribution to $B$ from the $\mu$ - $B \mu$ sector is

$$
\frac{\Delta B}{B_{\mathrm{AMSB}}} \sim \frac{\lambda^{2}}{4 N_{c} y_{t}^{2}} \frac{1}{(1+a \lambda / c)^{2}},
$$

so the anomaly-mediated contribution naturally dominates due to a combination of the large top Yukawa coupling, color factors, and order-1 factors.

\section{Phenomenology}

\subsection{Spectrum}

We now discuss the spectrum of the models under consideration. We consider the minimal model, with parameters

$$
F_{\varphi}, F, M, \mu, N_{\text {mess }}
$$

where $F_{\varphi}$ and $F$ are the anomaly- and gauge-mediated SUSY breaking parameters, $M$ is the messenger scale, and $N_{\text {mess }}$ is the number of $\mathbf{5} \oplus \overline{\mathbf{5}}$ messengers. We impose the unification condition eq. (2.18) to relate the doublet and triplet messengers. This means that the spectrum is qualitatively similar to gauge-mediated models, so we predict for example $m_{\tilde{q}} / m_{\tilde{\ell}_{R}} \sim \alpha_{3} / \alpha_{1}$. We can also relax this assumption and get spectra with (for example) colored and uncolored superpartner masses more closely degenerate [7]. We will not discuss this possibility here.

The messenger scale $M$ is fixed to be of order $F_{\varphi} \sim 50 \mathrm{TeV}$ in this model. The SUSY breaking terms generated at the messenger threshold are proportional to $F_{\varphi}$ and $F / M$ times a function of

$$
x=\frac{F}{M^{2}} .
$$

Positivity of messenger masses requires $x<1$. In practice, the dependence on $x$ is very weak unless $x=1$ to high accuracy (see appendix). This dependence therefore drops out. The SUSY breaking spectrum also depends on $M$ logarithmically through the renormalization group, since $M$ determines the scale at which we match the SUSY breaking masses onto the MSSM. For example, changing the messenger scale by a factor of 2 changes the spectrum at the level of a 1-loop correction, so this dependence can also be neglected. The result of this is that the SUSY breaking spectrum essentially depends only on the parameters

$$
F_{\varphi}, \frac{F}{M}, \mu, N_{\text {mess }} .
$$

After we fix the Higgs VEV to its observed value, the theory therefore depends on 2 continuous parameters, which we take to be

$$
M_{\mathrm{SUSY}} \equiv \frac{F_{\varphi}}{16 \pi^{2}}, \quad r \equiv \frac{F / M}{F_{\varphi}} .
$$

For a range of positive $r$ we obtain a physical solution with all scalar mass-squared terms positive, realistic electroweak symmetry breaking, and a neutralino LSP. In figure 1 we plot the Higgs mass and the LSP relic density for the minimal model with $N_{\text {mess }}=1$. 


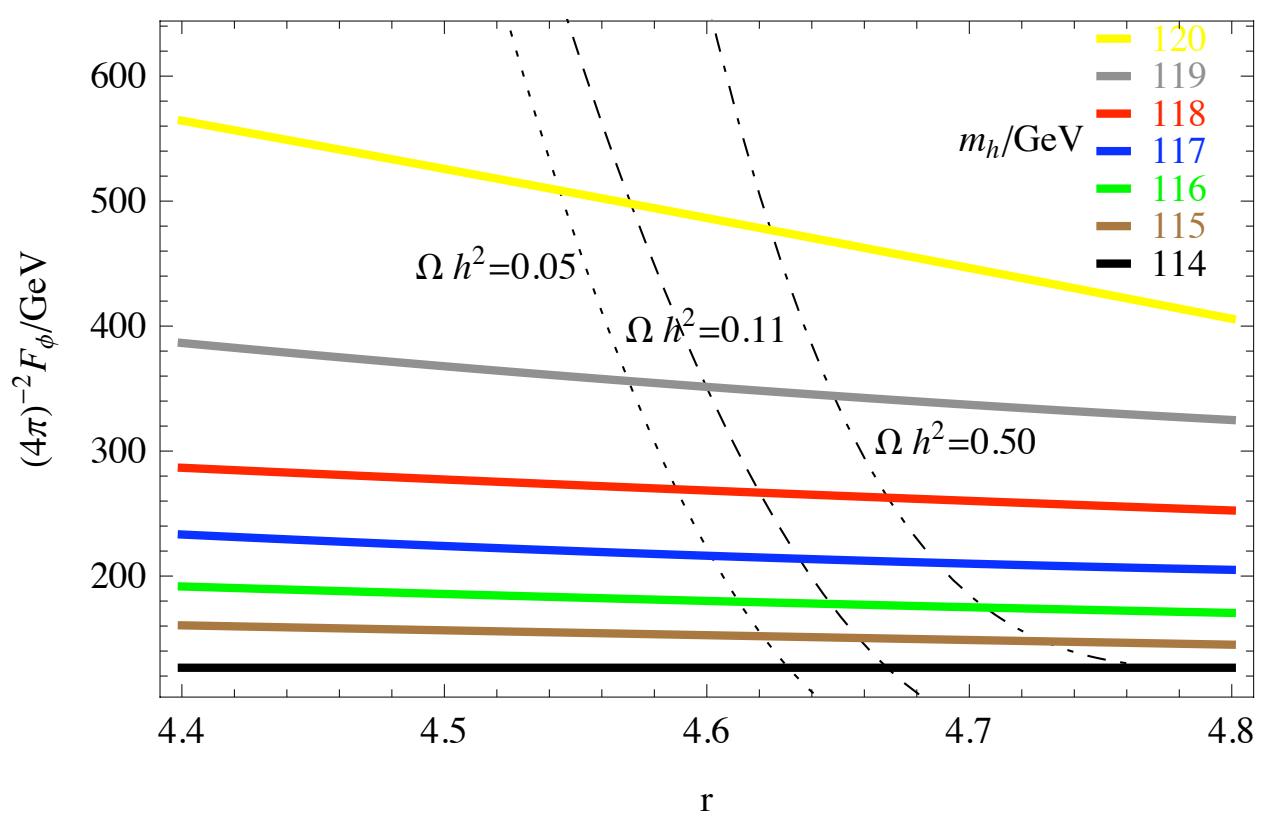

Figure 1. Lightest $C P$ even Higgs mass and neutralino LSP relic density in the minimal model with $N_{\text {mess }}=1$.

We find that electroweak symmetry breaking occurs only for $\mu>0$. These results were obtained using SuSpect [10]. The Higgs mass prediction is expected to be uncertain by about $\pm 2 \mathrm{GeV}$. We see that the Higgs mass is very close to the LEP bound, and the correct relic density can be obtained. The rest of the spectrum is shown in figures 2 and 3 for the models along the line where $\Omega h^{2}=0.11$.

\subsection{Dark matter}

The spin-independent direct detection dark matter-nucleon cross section for these models is plotted in figure 4. These results we obtained using DarkSUSY [11]. The cross sections are well below existing experimental bounds. Future ton-scale experiments such as LUX/ZEPLIN [12], SuperCDMS [13], and XENON1T [14] have an expected sensitivity to spin-independent cross sections in the range $10^{-46}$ to $10^{-48} \mathrm{~cm}^{2}$ for these masses, and will therefore be able to probe these models for WIMP masses up to approximately $200 \mathrm{GeV}$.

\subsection{Fine tuning}

These models have the usual fine-tuning problem of SUSY models. This arises because top and stop loops give a contribution to the Higgs quadratic term

$$
\Delta m_{H_{u}}^{2} \sim-\frac{N_{c} y_{t}^{2}}{4 \pi^{2}} m_{\tilde{t}}^{2} \ln \frac{M}{m_{\tilde{t}}} .
$$

The Higgs quadratic coupling must be of order the physical Higgs mass $m_{h^{0}}^{2}$, so a rough measure of the fine-tuning is therefore

$$
\text { tuning } \sim \frac{\Delta m_{H_{u}}^{2}}{m_{h^{0}}^{2}} .
$$




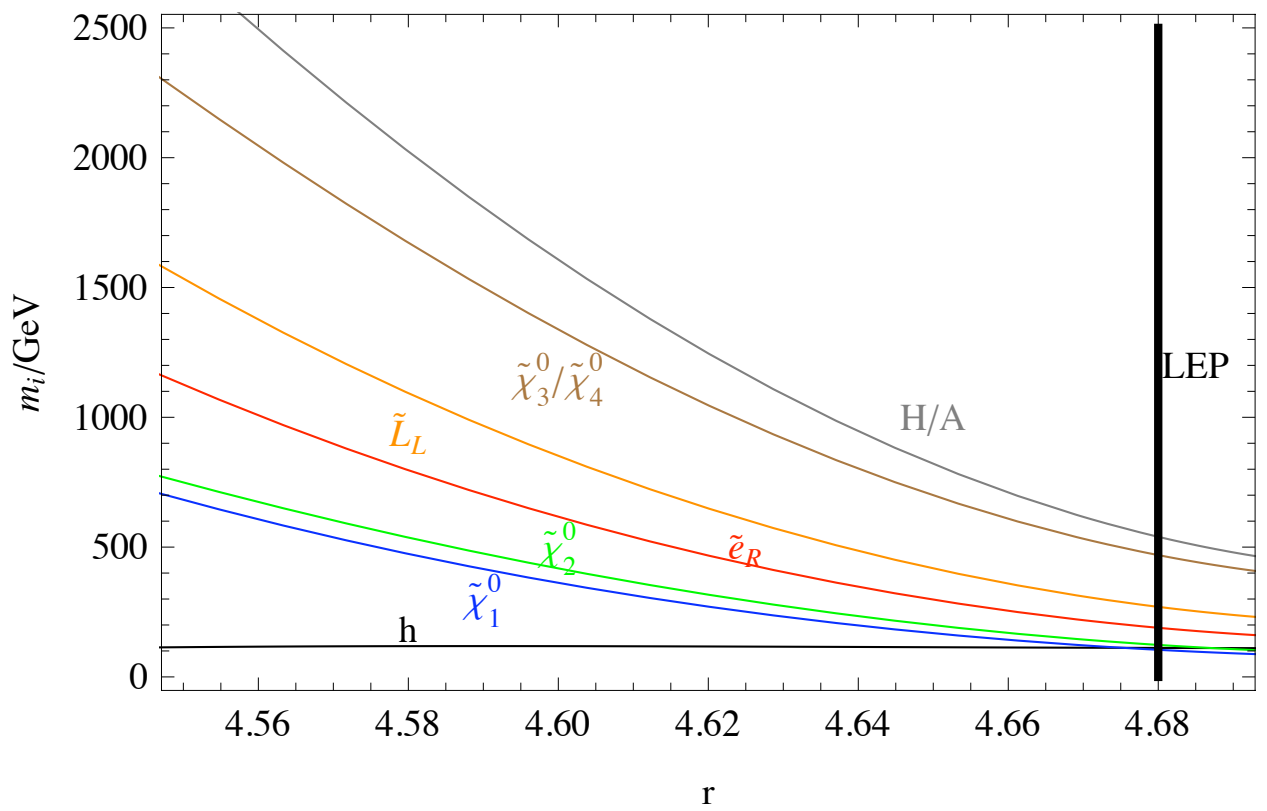

Figure 2. Uncolored superpartner masses in the minimal model for parameters where the LSP has the correct relic density.

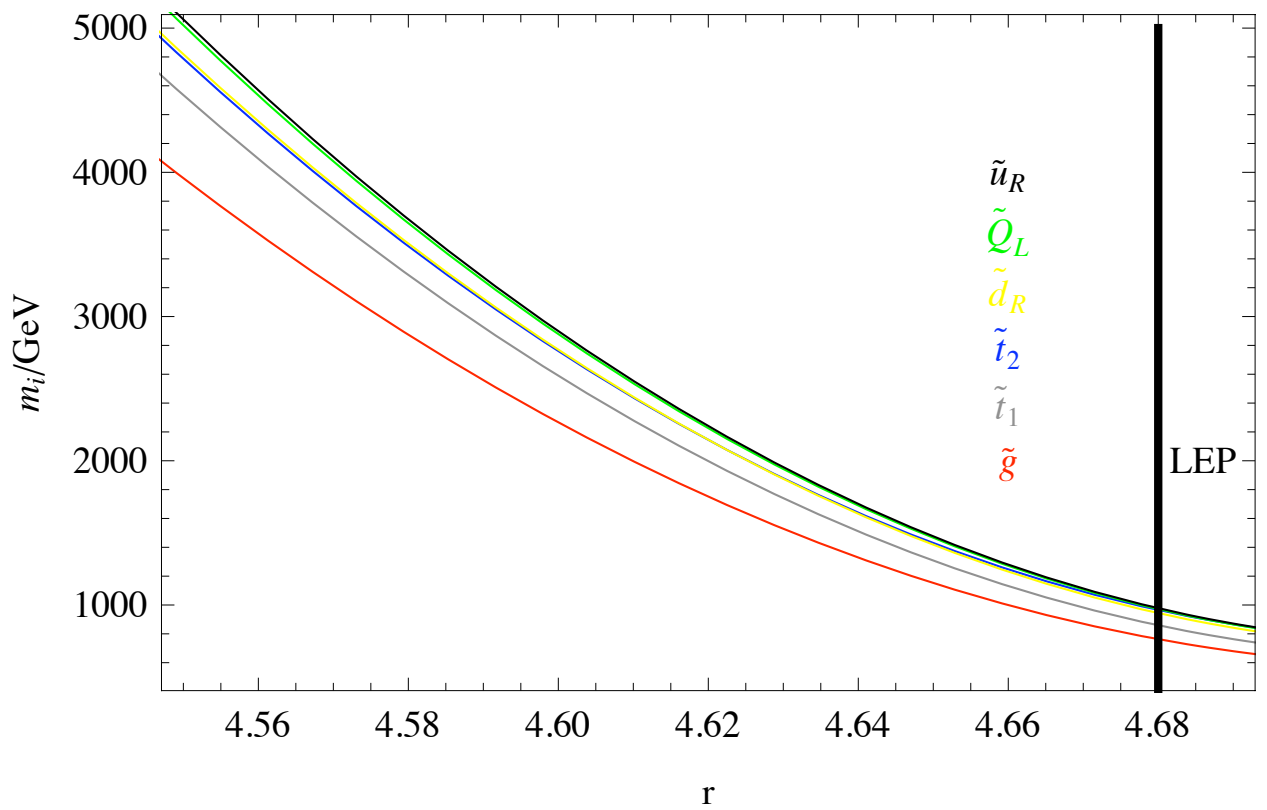

Figure 3. Colored superpartner masses in the minimal model for parameters where the LSP has the correct relic density.

This is $\sim 100$ in our models. We emphasize that this problem is shared by practically all other SUSY models. In particular, in models where the SUSY breaking terms are generated by standard model gauge interactions one always has

$$
\frac{m_{\tilde{t}}}{m_{\tilde{\ell}_{R}}} \sim \frac{\alpha_{3}}{\alpha_{1}} \sim 10,
$$




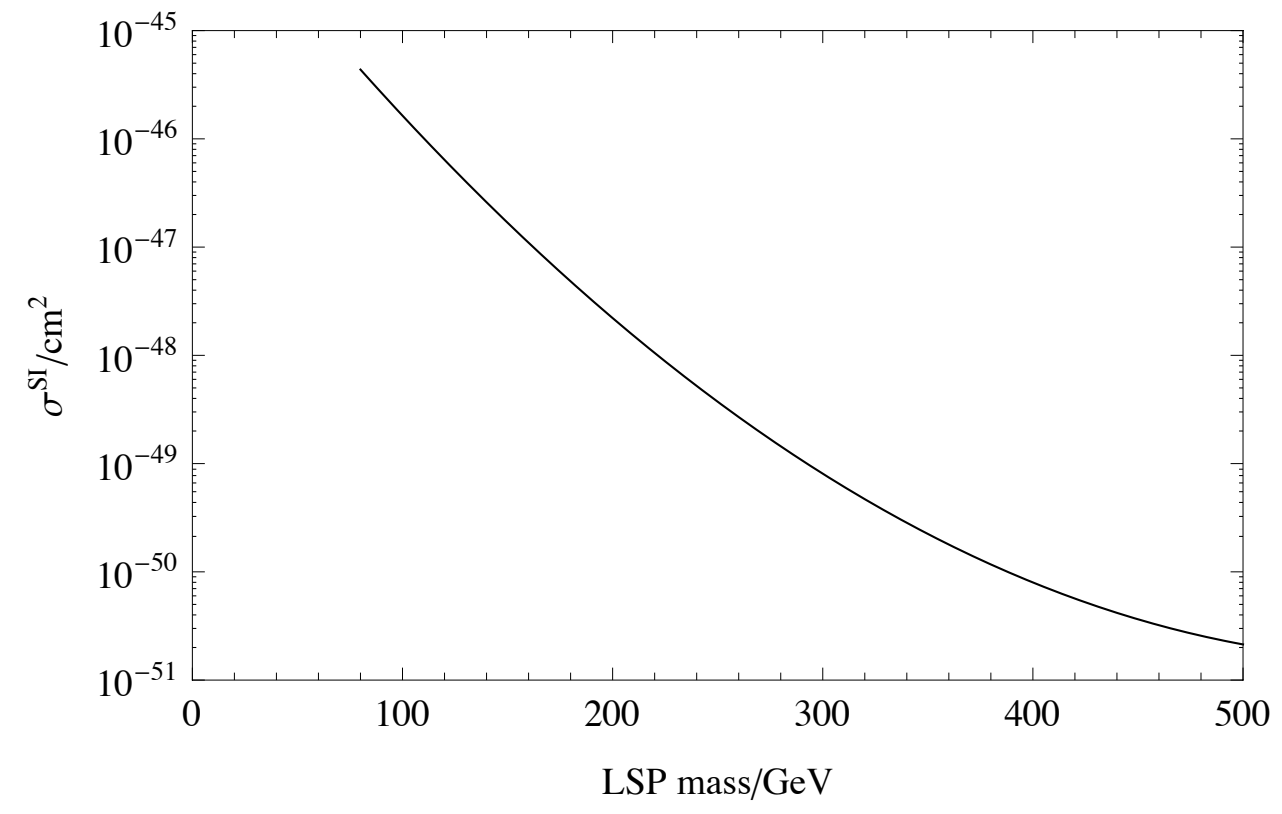

Figure 4. LSP direct detection cross section for minimal model with correct relic abundance.

and therefore $m_{\tilde{t}} \sim \mathrm{TeV}$. This implies that the model is tuned even without imposing the LEP bound on the Higgs mass.

There have been a number of solutions to the SUSY tuning problem proposed in the literature. One is exotic Higgs decays arising from additional structure in the Higgs sector. This may allow the Higgs to be light, but this will not significantly reduce the fine-tuning in models satisfying eq. (4.7). Another possibility is new contributions to the Higgs quartic coupling [15-17], which reduce the fine tuning by allowing larger Higgs quadratic terms. These generally affect only the Higgs sector, and the predictions for the squark, slepton, and gluino masses in this model still hold. Another possibility is that tuning may be the result of anthropic selection of a vacuum that breaks electroweak symmetry [18].

\section{Conclusions}

We have presented a minimal model of SUSY breaking that naturally solves the SUSY flavor problem, the $\mu$ problem, and has a viable WIMP dark matter candidate. SUSY is broken by anomaly mediation in the visible sector, with a messenger threshold at the scale $F_{\varphi} \sim 50 \mathrm{TeV}$ generated by holomorphic Kähler terms. Realistic $\mu$ and $B \mu$ terms are generated by a relaxation mechanism. The models are similar to gauge mediated models in that standard model gauge loops generate the SUSY breaking spectrum, and therefore we have for example $m_{\tilde{q}} / m_{\tilde{\ell}_{R}} \sim \alpha_{3} / \alpha_{1}$. However the origin of SUSY breaking in anomaly mediation gives some important differences with gauge-mediated models. First, the gravitino is naturally at the scale $F_{\varphi}$ so the LSP is automatically a WIMP candidate. Also, anomaly mediation gives rise to a $B$ term in the Higgs potential from the top loop, 
and this generally dominates the contribution from the sector that generates the $\mu$ term. This effectively eliminates one of the free parameters in this model.

The minimal model depends on only 2 continuous parameters, and the number of messengers. We have shown that this model can give realistic spectra with neutralino dark matter that can be detected in upcoming experiments. We believe these models deserve close study at the LHC.

\section{Acknowledgments}

We thank N. Setzer and S. Spinner for pointing out an error in the first draft. This work was partially carried out at the Aspen Center for Physics. This work was supported by DOE grant DE-FG02-91-ER40674.

\section{A SUSY breaking}

In this appendix we derive the results for the SUSY breaking terms at the scale $F_{\varphi}$. The new feature is the consistent combination of anomaly and gauge mediated contributions for the case $F / M \sim F_{\varphi}$ and $F / M^{2} \sim 1$.

The SUSY breaking induced by the threshold at the scale $F_{\varphi}$ is best understood by working in a formulation where all SUSY breaking is in the higher components of the mass $M$ and UV cutoff $\Lambda$. For gaugino masses, we write the effective theory below the scale $M$ as

$$
\mathcal{L}_{\text {eff }}=\int d^{2} \theta \operatorname{tr}\left(W^{\alpha} W_{\alpha}\right)\left[\tau(M, \Lambda)+a_{1} \frac{\bar{D}^{2} M^{\dagger}}{M^{2}}+a_{2}\left(\frac{\bar{D}^{2} M^{\dagger}}{M^{2}}\right)^{2}+\cdots\right]+\text { h.c. },
$$

where $\tau$ is the gauge coupling superfield, and the remaining terms are finite terms arising from integrating out the messengers at the scale $M$. Here $\tau, M$, and $\Lambda$ are all chiral superfields. We have

$$
\frac{\partial}{\partial \theta^{2}} \frac{\bar{D}^{2} M^{\dagger}}{M^{2}} \propto \frac{F^{2}}{M^{2}}
$$

so that the terms proportional to $a_{0}, a_{1}, \ldots$ are negligible for $F \gg M^{2}$. However, we are interested in the case $F \sim M^{2} \sim F_{\varphi}^{2}$, so we must include them to all orders. Terms such as $\bar{D}^{2} \Lambda^{\dagger} / \Lambda^{2}$ may also arise from integrating out physics at the cutoff, but these give a vanishing contribution to SUSY breaking in the limit $\Lambda \rightarrow \infty$. Mixed terms such as $\bar{D}^{2} \Lambda^{\dagger} / \Lambda M$ cannot arise because they violate decoupling of the scales $\Lambda$ and $M$, namely all terms that are unsuppressed as $\Lambda \rightarrow \infty$ must be nonsingular as $M \rightarrow 0$.

The contribution from $\tau$ can be computed using the techniques of refs. [19, 20]:

$$
\frac{\partial \tau}{\partial \theta^{2}}=\left(F_{\varphi} \frac{\partial}{\partial \ln \Lambda}+\frac{F}{M} \frac{\partial}{\partial \ln M}\right) \tau
$$

We evaluate these derivatives at a scale $\mu<M$. In this way we obtain the $\tau$ contribution to the gaugino mass at the scale $M$ :

$$
m_{1 / 2}(M)=\frac{1}{g}\left[-F_{\varphi} \beta_{g}^{\prime}+\frac{F}{M}\left(\beta_{g}^{\prime}-\beta_{g}\right)\right]+\cdots
$$


The limit $F / M \rightarrow F_{\varphi}$ corresponds to a superpotential mass term, and gives

$$
m_{1 / 2} \rightarrow \frac{F_{\varphi}}{g} \beta_{g}+\cdots
$$

which is the anomaly-mediated contribution computed in the effective theory below the scale $M$. This is the famous decoupling of thresholds in anomaly mediation. This decoupling can be viewed as a consequence of the identity

$$
\frac{\partial}{\partial \ln \Lambda}+\frac{\partial}{\partial \ln M}+\frac{\partial}{\partial \ln \mu}=0
$$

where $\mu$ is the renormalization scale. In the limit of an explicit superpotential mass term $F / M \rightarrow F_{\varphi}$ this gives

$$
\frac{\partial}{\partial \theta^{2}} \rightarrow-F_{\varphi} \frac{\partial}{\partial \ln \mu},
$$

so the gaugino mass arising from $\tau$ is given by the anomaly-mediated contribution in the effective theory in this limit.

This decoupling is violated by the higher order terms $a_{1}, a_{2}, \ldots$ in eq. (A.1). These involve higher powers of

$$
x=\frac{F}{M^{2}}
$$

and correspond to computing the gauge-mediated contribution to all orders in $x$. This calculation was performed in refs. [21, 22], giving the result

$$
m_{1 / 2}(M)=\frac{1}{g}\left[-F_{\varphi} \beta_{g}^{\prime}+\frac{F}{M}\left(\beta_{g}^{\prime}-\beta_{g}\right) G(x)\right],
$$

where

$$
\beta_{g}=\frac{d g}{d \ln \mu}
$$

is the beta function for the gauge coupling $g$ in the effective theory below the scale $M$, and the primed quantities refer to the theory above the scale $M$. The function $G$ is given by

$$
G(x)=\frac{1}{x^{2}}[(1+x) \ln (1+x)+(1-x) \ln (1-x)] .
$$

For $x \rightarrow 0, G(x) \rightarrow 1$, while for $x \rightarrow 1, G(x) \rightarrow \ln 4 \simeq 1.4$.

The scalar masses are a bit more subtle because there are in general mixed gauge- and anomaly-mediated contributions. The effective theory below the scale $M$ contains terms

$$
\mathcal{L}_{\text {eff }}=\int d^{4} \theta Q^{\dagger} Q\left[Z(|M|,|\Lambda|)+c_{1}\left|\frac{D^{2} M}{M^{2}}\right|^{2}+c_{2}\left|\frac{D^{2} M}{M^{2}}\right|^{4}+\cdots\right],
$$

where $Z$ is the wavefunction renormalization constant. Here $Z,|\Lambda|=\left(\Lambda^{\dagger} \Lambda\right)^{1 / 2}$, and $|M|=$ $\left(M^{\dagger} M\right)^{1 / 2}$ are real superfields. Again, contributions involving e.g. $D^{2} \Lambda / \Lambda M$ are absent. The contribution from $Z$ can be computed from

$$
\frac{\partial Z}{\partial \theta^{2}}=\frac{1}{2}\left(F_{\varphi} \frac{\partial}{\partial \ln |\Lambda|}+\frac{F}{M} \frac{\partial}{\partial \ln |M|}\right) Z
$$


and we obtain for the scalar mass

$$
\begin{aligned}
& m_{0}^{2}(M)=-\frac{\partial}{\partial \bar{\theta}^{2}} \frac{\partial}{\partial \theta^{2}} \ln Z \\
&=-\frac{1}{4}\left\{F_{\varphi}^{2} \frac{\partial \gamma^{\prime}}{\partial g_{i}^{\prime}} \beta_{i}^{\prime}-2 F_{\varphi} \frac{F}{M}\left(\frac{\partial \gamma^{\prime}}{\partial g_{i}^{\prime}}-\frac{\partial \gamma}{d g_{i}}\right) \beta_{i}^{\prime}\right. \\
&\left.\quad-\left(\frac{F}{M}\right)^{2}\left[\frac{\partial \gamma}{\partial g_{i}}\left(\beta_{i}^{\prime}-\beta_{i}\right)-\left(\frac{\partial \gamma^{\prime}}{d g_{i}^{\prime}}-\frac{\partial \gamma}{\partial g_{i}}\right) \beta_{i}^{\prime}\right]\right\}+\cdots
\end{aligned}
$$

where $g_{i}$ are the couplings in the theory and $\gamma=d \ln Z / d \ln \mu$ is the anomalous dimension of the scalar field. We can again check that for $F / M \rightarrow F_{\varphi}$ this reduces to the anomalymediated contribution computed in the effective theory below the scale $M$.

The terms proportional to $c_{1}, c_{2}, \ldots$ in eq. (A.12) give corrections that are higher order in $x$, corresponding to the full gauge-mediated contribution. Thus we obtain the full contribution

$$
\begin{aligned}
m_{0}^{2}(M)=-\frac{1}{4}\{ & F_{\varphi}^{2} \frac{\partial \gamma^{\prime}}{\partial g_{i}^{\prime}} \beta_{i}^{\prime}-2 F_{\varphi} \frac{F}{M}\left(\frac{\partial \gamma^{\prime}}{\partial g_{i}^{\prime}}-\frac{\partial \gamma}{d g_{i}}\right) \beta_{i}^{\prime} \\
& \left.\quad-\left(\frac{F}{M}\right)^{2}\left[\frac{\partial \gamma}{\partial g_{i}}\left(\beta_{i}^{\prime}-\beta_{i}\right)-\left(\frac{\partial \gamma^{\prime}}{d g_{i}^{\prime}}-\frac{\partial \gamma}{\partial g_{i}}\right) \beta_{i}^{\prime}\right] H(x)\right\},
\end{aligned}
$$

where

$$
H(x)=\frac{1+x}{x^{2}}\left[\ln (1+x)-2 \operatorname{Li}_{2}\left(\frac{x}{1+x}\right)+\frac{1}{2} \operatorname{Li}_{2}\left(\frac{2 x}{1+x}\right)\right]+(x \leftrightarrow-x) .
$$

The absence of corrections to the mixed terms is due to the fact that there are no corrections of the form $D^{2} \Lambda / \Lambda M$ in eq. (A.12). For fields that do not have tree-level couplings to the messengers, we have $\gamma^{\prime}=\gamma$, and the mixed terms vanish.

Open Access. This article is distributed under the terms of the Creative Commons Attribution Noncommercial License which permits any noncommercial use, distribution, and reproduction in any medium, provided the original author(s) and source are credited.

\section{References}

[1] L. Randall and R. Sundrum, Out of this world supersymmetry breaking, Nucl. Phys. B 557 (1999) 79 [hep-th/9810155] [SPIRES].

[2] G.F. Giudice, M.A. Luty, H. Murayama and R. Rattazzi, Gaugino Mass without Singlets, JHEP 12 (1998) 027 [hep-ph/9810442] [SPIRES].

[3] S. Kachru, L. McAllister and R. Sundrum, Sequestering in string theory, JHEP 10 (2007) 013 [hep-th/0703105] [SPIRES].

[4] A. Pomarol and R. Rattazzi, Sparticle masses from the superconformal anomaly, JHEP 05 (1999) 013 [hep-ph/9903448] [SPIRES].

[5] R. Sundrum, 'Gaugomaly' mediated SUSY breaking and conformal sequestering, Phys. Rev. D 71 (2005) 085003 [hep-th/0406012] [SPIRES]. 
[6] A.E. Nelson and N.T. Weiner, Extended anomaly mediation and new physics at 10-TeV, hep-ph/0210288 [SPIRES].

[7] K. Hsieh and M.A. Luty, Mixed gauge and anomaly mediation from new physics at 10-TeV, JHEP 06 (2007) 062 [hep-ph/0604256] [SPIRES].

[8] N. Seiberg, Naturalness Versus Supersymmetric Non-renormalization Theorems, Phys. Lett. B 318 (1993) 469 [hep-ph/9309335] [SPIRES].

[9] G.R. Dvali, G.F. Giudice and A. Pomarol, The $\mu$-Problem in Theories with Gauge-Mediated Supersymmetry Breaking, Nucl. Phys. B 478 (1996) 31 [hep-ph/9603238] [SPIRES].

[10] A. Djouadi, J.-L. Kneur and G. Moultaka, SuSpect: A Fortran code for the supersymmetric and Higgs particle spectrum in the MSSM, Comput. Phys. Commun. 176 (2007) 426 [hep-ph/0211331] [SPIRES].

[11] P. Gondolo et al., DarkSUSY: Computing supersymmetric dark matter properties numerically, JCAP 07 (2004) 008 [astro-ph/0406204] [SPIRES].

[12] D.N. McKinsey et al., The Lux Dark Matter Search, J. Phys. Conf. Ser. 203 (2010) 012026 [SPIRES].

[13] D.S. Akerib et al., The SuperCDMS proposal for dark matter detection, Nucl. Instrum. Meth. A 559 (2006) 411 [SPIRES].

[14] E. Aprile, XENON1T: a ton scale LXe dark matter experiment talk at UCLA Dark Matter 2010 Conference, Marina del Rey, California U.S.A., February 24-26 2010, http://www.physics.ucla.edu/hep/dm10/presentations.html.

[15] P. Batra, A. Delgado, D.E. Kaplan and T.M.P. Tait, The Higgs mass bound in gauge extensions of the minimal supersymmetric standard model, JHEP 02 (2004) 043 [hep-ph/0309149] [SPIRES].

[16] R. Harnik, G.D. Kribs, D.T. Larson and H. Murayama, The minimal supersymmetric fat Higgs model, Phys. Rev. D 70 (2004) 015002 [hep-ph/0311349] [SPIRES].

[17] S. Chang, C. Kilic and R. Mahbubani, The new fat Higgs: Slimmer and more attractive, Phys. Rev. D 71 (2005) 015003 [hep-ph/0405267] [SPIRES].

[18] G.F. Giudice and R. Rattazzi, Living dangerously with low-energy supersymmetry, Nucl. Phys. B 757 (2006) 19 [hep-ph/0606105] [SPIRES].

[19] G.F. Giudice and R. Rattazzi, Extracting Supersymmetry-Breaking Effects from Wave-Function Renormalization, Nucl. Phys. B 511 (1998) 25 [hep-ph/9706540] [SPIRES].

[20] N. Arkani-Hamed, G.F. Giudice, M.A. Luty and R. Rattazzi, Supersymmetry-breaking loops from analytic continuation into superspace, Phys. Rev. D 58 (1998) 115005 [hep-ph/9803290] [SPIRES].

[21] S. Dimopoulos, G.F. Giudice and A. Pomarol, Dark matter in theories of gauge-mediated supersymmetry breaking, Phys. Lett. B 389 (1996) 37 [hep-ph/9607225] [SPIRES].

[22] S.P. Martin, Generalized messengers of supersymmetry breaking and the sparticle mass spectrum, Phys. Rev. D 55 (1997) 3177 [hep-ph/9608224] [SPIRES]. 